\title{
Computational Prometheus: How Reinforcement was stolen, and how to steal it back.
}

\author{
Greg Jensen ${ }^{1}$ and David Freestone ${ }^{2}$ \\ ${ }^{1}$ Dept. of Psychology, Columbia University \\ ${ }^{2}$ Dept. of Psychology, William Paterson University
}

\begin{abstract}
There are now tools and techniques in computer science that make building and testing complicated process models of behavior easy. One such approach to process models, "Reinforcement Learning," has found a second home in neuroscience. When fit to animal data, these process models give trial-by-trial estimates of the animal's changing associations as it learns. At the same time, new computational tools are emerging that make it easier than ever to fit complex models to large, hierarchical data sets. However, before behavior analysts can fully embrace this new set of tools, they will need to reconcile with its divergent terminology. On the one hand, the scope of 'reinforcement' in behavior analysis has broadened to include more properties of the reinforcer. Meanwhile, the term's usage in computer science (and thus increasingly in neuroscience) is much less theoretically constrained than has been traditional in behavior analysis. It is now time to take stock of the new tools, their successes and limitations, and what role behavior analysis now has to play. Here, we sketch a reinforcement learning model of matching behavior, and use it to show how these new computational tools can help our search for the mechanisms of behavior.
\end{abstract}

Keywords: Reinforcement Learning, Generalized Matching, Behavior Analysis, Rats

Ten years ago, the late Edmund Fantino wrote a commentary entitled, "Behavior analysis: Thriving, but how about its future?" In it, he called on the torch-bearers of behavior analysis to pursue interdisciplinary collaborations:

I still believe we should be doing more in the way of addressing traditional problems of interest in other areas, including cognitive, developmental, social, and physiological psychology. I believe we have been rewarded when we have taken a more interdisciplinary approach to the study of behavior.

-Fantino (2008)

Although optimistic in its tone, Fantino was speaking to the insularity of modern behavior analysis and voicing a concern that the world would pass behavior analysis by if it made no inroads into related fields. This concern is not new. Jack Michael's (1980) presidential address to the Association of Behavior Analysis, nearly forty years ago, was titled "Flight from behavior analysis."

Meanwhile, both reports in the popular press and an overview of scholarly works would seem to suggest a resurgence of behavior analysis. The proportion of scholarly works (indexed by Google Scholar) that include the word 'reinforcement' has been growing since the 1950s (Figure 1, left). Admittedly, this is a crude measure, as some of these articles related to construction materials, but reinforcement's special significance in the language of behaviorism as one of its chief linguistic bulwarks against mentalist theorizing ensures that nearly all major works in both the Skinnerian and Pavlovian traditions are included. 
Use of "Reinforcement"

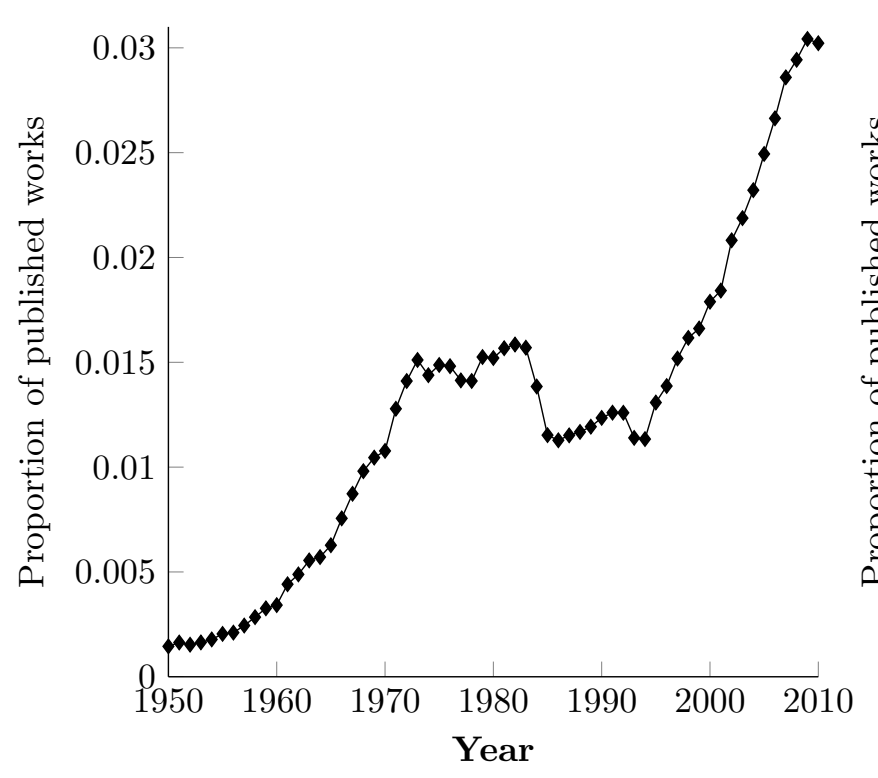

Use of Specific Terminology

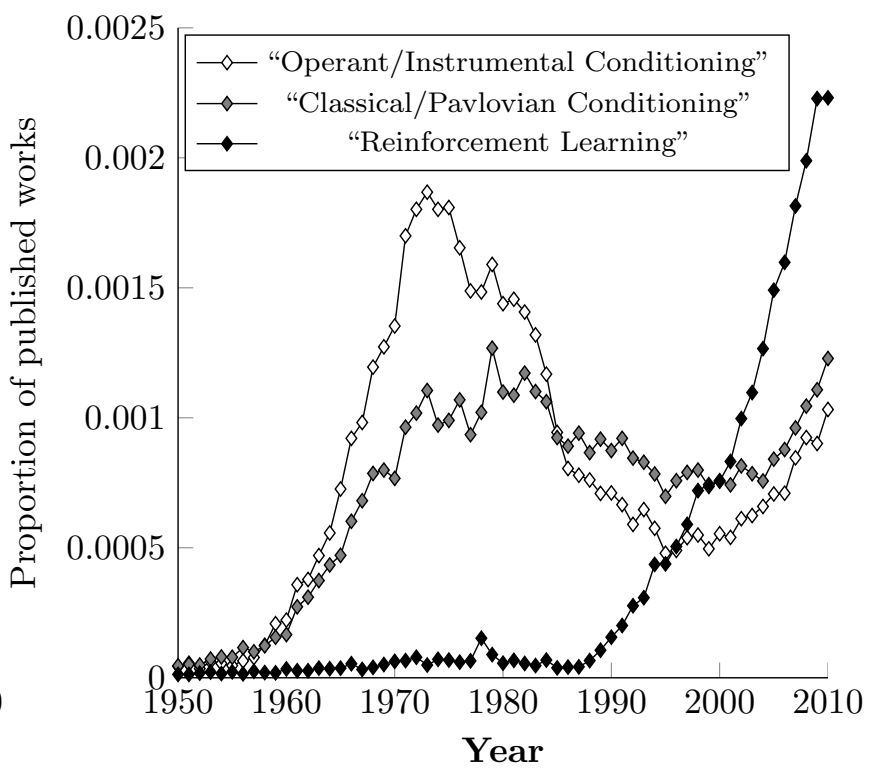

Figure 1. Proportions of published academic works using behavior-analytic terminology over time, according to Google Scholar. (Left) Proportion of works using the word "reinforcement" in any context. (Right) Proportion of works using the phrases "operant conditioning" and/or "instrumental conditioning" (in white), those using "classical conditioning" or "Pavlovian conditioning" (in gray), and those using "reinforcement learning" (in black).

Most behavior analysts would recognize the 'flight' in the 1980s, but many will be surprised to see that 'reinforcement' is now used twice as often as it was at the height of behaviorism's popularity.

To better understand the disconnect between the apparent flight from behavior analysis and the resurgent popularity of 'reinforcement,' a more fine-grained analysis is revealing. As shown in Figure 1 (right), usage of both 'operant conditioning' and 'classical conditioning' conditioning peaked in the early 1970s ('instrumental conditioning' and 'Pavlovian conditioning' are also included in this analysis). Reinforcement's recent resurgence is driven almost entirely by the phrase 'reinforcement learning.' As a phrase, reinforcement learning is almost unheard of prior to the mid-1980s. Since then, its meteoric rise continues to accelerate. Reinforcement Learning (RL) is the reason we are seeing reinforcement in the news, but the term has no grounding in the behavior analysis of the 1960s and 70s. In fact, the term is not even psychological. Reinforcement learning is a branch of the "machine learning" field in computer science.

Despite the wide gap between them, we view behavior analysis and reinforcement learning as addressing complementary problems. Behavior analysts study behavior experimentally, often without a precise process model, and in doing so have found key phenomena of behavior that any complete process model must explain. Reinforcement learning, on the other hand, studies process models of a particular form, but has placed less emphasis on explaining the behavior of organisms, focusing instead on solving difficult problems efficiently. Behavior analysts and computer scientists are accustomed to asking such different questions that it may not initially be clear what each can offer to the other.

Below, we first discuss what we believe are the strengths and weaknesses of the behavior analysis and $\mathrm{RL}$ approaches to studying and building mechanisms for action. Then, as an example of how reinforcement learning can enhance behavior analysis, we use a simple process model to analyze generalized matching data from an experiment using four operanda. We show that the reinforcement learning model makes important features of behavior clear that traditional generalized matching analyses would have obscured. 
We end by discussing where we, as a community, go from here.

\section{A Terminological Land-Grab}

In the 1970s, the study of artificial intelligence was at its nadir. Efforts to build the "electronic brains" dreamed of by pioneers like Turing and von Neumann had floundered. Problems that were once considered foundational and presumed to be trivial (such as semantic networks and computer vision) were proving to be far more difficult than anticipated, and funding was drying up (Lenat, 2008). It was in this atmosphere that "artificial intelligence" gave way to the more agnostic "machine learning" approach, in which expert systems were designed to solve practical problems, without much regard for whether they solved problems in the same way that biological intelligence might. As computing spread rapidly in the private sector, so too did the design of these expert systems, and the study of their underlying "machine learning" algorithms. Over the subsequent 20 years, this explosion of research culminated in Sutton and Barto's Reinforcement Learning: An Introduction (1998), which remains one of the field's most important texts. As of this writing, it has been cited over 28,000 times in academic research.

What is striking about Sutton and Barto's book is that despite using the word "reinforcement" to describe a mechanism of learning, the text makes almost no reference to behavior analysis, or indeed psychology of any kind. Skinner, Pavlov, and Hull are each cited once, and then only in endnotes that make no mention of the content of their theories. Rescorla and Wagner are not mentioned at all. Insofar as reinforcement is discussed as a psychological principle, that discussion is limited to the ideas of Thorndike.

The reason for these omissions is that "reinforcement learning" (abbreviated RL) is not a theory of learning in organisms. Instead, it is a broad class of computer algorithms that are used to make decisions or inferences. The chief distinction between RL and other forms of machine learning is that RL makes inferences based on incomplete information about the problem space. RL algorithms, for example, typically do not know what every possible outcome of every possible decision might be. To solve such problems, RL algorithms define some "value function" that converts measurements of behavior and outcomes into a generic value score, and then seek to maximize that score over projected future behaviors. That score is then the input for a "policy algorithm" that select future behaviors. It is this tradition of $\mathrm{RL}$, and not the theories of reinforcement from 20th century psychology, that has come to dominate the biomedical sciences, as well as making headlines in what is being called a new era of AI.

As computer science evolved into a field in its own right, it enjoyed very little cross-talk with psychology prior to the cognitive revolution. Throughout these early years, psychology's theories of learning were raised in RL papers from time to time, but almost always in a superficial way. In a pivotal paper, Sutton and Barto (1981) point out the nice mathematical properties of the Rescorla-Wagner model (1972), but they also point out that a mathematically identical formulation was first proposed, and more rigorously described, by Widrow and Hoff (1960). Seventeen years later, RL's bible would omit Rescorla and Wagner entirely in favor of Widrow and Hoff's formulation. This pattern is typical in the literature. After all, behavior analysts have historically found the behavior of organisms to be more important than the behavior of mathematical abstractions, and consequently RL as a branch of applied mathematics has little to learn from them.

Although both fields use the term "reinforcement" to describe how agents learn, that might be as far as the similarities go. This view, however, neglects the middle ground occupied by computational neuroscience, a field that has drawn heavily from the tools of applied mathematics but is also motivated to constrain those tools in ways that are biologically and behaviorally plausible. Psychology can benefit from applying these new tools insofar as they motivate theories and new experiments. At the same time, computational neuroscientists can benefit from psychology's applications of its discoveries, because a mismatch between model and experiment can only be uncovered by experimentalists. RL tools can help 
us ask new questions, and we can help illuminate the gaps in the new models that result.

Our view is that behavior analysis and computational neuroscience hone in on how organisms learn, but in different ways. While behavior analysts tend to examine behavior in order to constrain possible future models, computational methods begin with simple models, designed according to first principles, such as providing an approximation to dynamic programming (Bellman, 1957). These models are always imperfect but often capture some essential characteristics of behavior. A model can then be refined to reduce the gap between its predictions and the empirical results. A behavior analyst may ask how behavior on some task limits what models are plausible, and a computational neuroscientist may ask how well a particular model performs on real tasks. Below, we describe the strengths of the two approaches, and then because our readers are more familiar with the behavior analyst approach, we give a case study in the reinforcement learning approach. The code and data are available from an Open Science Foundation repository ( https://osf.io/rt54h/ ).

\section{What Behavior Analysis Does Well}

The tenet of behaviorism that still rings as true today as it did at the height of its influence is that we have no immediate access to how the mind, or something like it, controls behavior.

When we attribute behavior to a neural or mental event, real or conceptual, we are likely to forget that we still have the task of accounting for the neural or mental event. When we assert that an animal acts in a given way because it expects to receive food, then what began as the task of accounting for learned behavior becomes the task of accounting for expectancy. The problem is at least equally complex and probably more difficult.

-Skinner (1950)

Since a behavior analyst's goal is to predict and modify behavior, there has been considerable caution about defining terms like "reinforcement" with respect to a specific internal mechanism. Internal states are real, and unavoidable, but they are concealed beneath the behavior. Many different internal models may predict the same behavior, and applied behavior analysis is more concerned with making good predictions than it is with anointing any single model as "true" (Staddon and Zanutto, 1998). From a behavior analyst's perspective, a precise definition of an internal variable like association is less important than determining its properties.

Nevertheless, Thorndike $(1898,1911)$ and others gave verbal descriptions of what reinforcement does: It acts in exactly the same way as construction materials do. It lays the foundation and strengthens an association between a stimulus and a response. An association is a semantic tool that sits between learning and performance, not quite a model. It does not need a precise definition because it is a label of an internal variable, rather than a definition. Cognitive psychologists have criticized this (Gallistel, 2007), and they have a point, but there is also strength in this approach. Approximate verbal descriptions let us speak coherently about a set of results while also keeping the space of possible models as large as possible for as long as possible, until some data constrains it. Behavior analysts move from the outside in.

The Rescorla-Wagner model breaks from this tradition. By the early 1970s, a host of empirical results put constraints on what properties associations could have. Blocking had just been established (Kamin, 1969); a reinforcer's effectiveness in modifying an association - regardless of what those terms mean depends on a complex history of reinforcement. In cue combination experiments, two different stimuli were paired with the same reinforcer on different trials, then presented as a compound (Rescorla and Wagner, 1972; Kalafut and Church, 2017). The animal responded more to the compound than to either stimulus alone, suggesting behavior is the output of some integration process over individual associations. 
Associations, whatever they are, combine. Many theorists, from Hull (1943) to Solomon (1980), have suggested that the summation of associations for each stimulus gives rise to a single associative strength that governs the responses. The Rescorla-Wagner (RW) model was an attempt to build a process model, updating associations after each trial, that accounted for these results and unified them. Echoing the BushMosteller model (1955), an association (here denoted as $Q$ ) should be updated after each reinforcement or nonreinforcement as follows:

$$
Q_{s, t+1} \leftarrow Q_{s, t}+\alpha\left(r_{t}-\sum_{s} Q_{s, t}\right)
$$

Here, $\alpha$ is the learning rate (the model's only free parameter) describing how much the association changes after reinforcement. Each stimulus has its own associative strength (indexed by s), and their sum creates a prediction about the reinforcement, $\sum_{s} Q_{s, t}$. Response rate is assumed to be a readout of this prediction, so this model predicts cue combination. The discrepancy between the reinforcement and the prediction $\left(r_{t}-\sum_{s} Q_{s, t}\right)$ matches Kamin's idea of surprise (Kamin, 1969), so it handles blocking. Crucially, the RW model not only explained existing data, but its specificity also enabled new predictions, which in turn motivated new lines of experimentation (Rescorla, 1971; Siegel and Allan, 1996). Some of these predictions have been confirmed by experiment, while others have been rejected (Miller et al., 1995). It has been highly influential for guiding new experiments. Its successes have helped constrain the concept of an association and reinforcements role in it. Its failures have, too.

Today, an association is a richer concept than radical behaviorists intended. When a stimulus is presented, animal behavior reflects the delay to food (Mazur, 2006), the magnitude of the upcoming food (Morris and Bouton, 2006), and intertrial interval (Bouton and Hendrix, 2011). Scrub jay caching and retrieval behavior reflects the what-where-when of the cached item (Clayton and Dickinson, 1998; Dally et al., 2006). The Temporal Coding Hypothesis pushes in this direction, too, suggesting animals can retrieve several memories associated with the reinforcement simultaneously (Savastano and Miller, 1998; Arcediano and Miller, 2002). The value of having a catch-all term like association for the hidden layer that separates memory and behavior is in its flexibility to shrink and grow as our data constrain the list of possible models.

Despite the RW model's success in motivating avenues of research, behavior analysts remain skeptical of specific models, preferring to keep the space of possible models large. As far as we can tell, the reason behavior analysts avoid terms like "representation" is because it posits a mechanism — perhaps an internal symbolic language with a set of operations that transform those symbols, or alternatively a computational framework that measures and manipulates variables. Any specific representational proposal feels as though it limits the model space, constraining it more than is needed. Behavior analysts, never able to peek inside the head, generally prefer to take an outside-in approach and focus on discovering the rules that govern behavior.

\section{What Computer Science Does Well}

Computer scientists who study machine learning create abstract agents that learn from input data. Abstraction is useful because a single general algorithm could potentially be used in different machines with different peripheral devices, that is, different sensory systems and behaviors. Computer scientists build from the inside out. They design the hidden layer, the intervening variable, first. Computational neuroscientists work largely the same way, except that they hope their models are constrained by biological plausibility. 
Under an RL framework, algorithms (or agents) select from a set of behaviors (or actions) in various contexts (or states), as a function of prior experience. The analyst must specify the formal rules for describing how agents transition from one state to the next, which may or may not be influenced by actions. Additionally, rules must be specified that define a value function. Value is gained or lost as the agent transitions from one state to the next, and the agent's goal is to behave in a way that maximizes its value. This is all brought together under some action policy, which defines how the agent converts its value into actions. Any algorithm that satisfies these criteria, no matter how complex, falls under the RL umbrella. Because each of these pieces needs to be fully specified, every RL algorithm, by definition, is able to generate simulated behavior.

$\mathrm{RL}$ is strictly agnostic about mentalistic language and labels. Only the equations matter. Cognitive concepts like representation are welcome, so long as they are defined precisely enough to specify value functions and action policies. Consequently, the tools developed by the RL field have been enormously popular in cognitive and computational neuroscience.

This is not to say, however, that RL is necessarily cognitive. Because RL algorithms are developed as flexible tools, rather than as theories of the natural world, they can be pressed into service by scientists who can then impose whatever constraints they think are appropriate. They behave more like statistical procedures, which can provide succinct descriptions given a set of assumptions. After all, the RescorlaWagner model is just an iterative way of computing a weighted average. But RL algorithms aren't quite like classical statistics, either, because of their emphasis on process, rather than on summary description. Formally, most statistical procedures familiar to psychologists assume the independence (or, at least, the exchangeability) of observations, whereas RL models depend critically on their sequential interdependence.

The field of reinforcement learning progresses by building better models only as needed. They encounter a situation where their current RL algorithms fail, and then modify the algorithm to fix it. For example, algorithms have been modified to learn more quickly (Azar et al., 2011), generalize to different tasks (Silver et al., 2018), adapt abruptly (Wang et al., 2017), and implement a hierarchy of goals that are simultaneously pursued by lower level action plans (Ribas-Fernandes et al., 2011). These extensions are out of necessity. Fix what fails.

Behavior analysts shrink the model space from the outside in, computer scientists extend a precise algorithm from the inside out. One views the forest from afar, while the other closely examines individual trees. Behavior analysis needs to occasionally take stock of which models are now plausible given the constraints and view what a specific model with those constraints would look like. Early on, the Rescorla-Wagner did this with incredible success. But in our opinion, behavior analysis does not draw its inspiration from precise models often enough.

To give an idea of how RL can be used in behavior analysis, we turn to a topic for which we have a long-standing interest: Choice allocation under concurrent schedules. Our goal is first to show how RL algorithms tell us something about matching behavior that we could not know from simple summaries of behavior (like generalized matching). Second, our goal is to show how to implement reinforcement learning models on a fairly complex dataset using modern statistical software. The result is a joint distribution of parameter estimates, from which we can compute any statistical summary we desire.

\section{A Worked Example: Generalized Matching}

Generalized matching specifies a precise relationship between the relative reinforcement rates on several options and how much animals spend their time foraging at those options. When reinforcers became available at unpredictable times, the proportions of choices between operanda $a, b, c$, and so forth is well described by generalized matching: 


$$
\frac{B_{a}}{\kappa_{a}\left(R_{a}\right)^{s}}=\frac{B_{b}}{\kappa_{b}\left(R_{b}\right)^{s}}=\frac{B_{c}}{\kappa_{c}\left(R_{c}\right)^{s}}=\ldots
$$

Here, $\kappa_{a}$ is a bias term, which is generally taken to represent features of the operandum. For example, $\kappa_{a}$ will be small if it takes more effort to manipulate the operandum. The variable $s$ describes how sensitive the animal is to the relative rates of reinforcement across all options.

Generalized matching is an extension of Herrnstein's matching law (Herrnstein, 1961), and was originally formulated by Baum (1974) as:

$$
\frac{B_{a}}{B_{b}}=\frac{\kappa_{a}}{\kappa_{b}}\left(\frac{R_{a}}{R_{b}}\right)^{s}
$$

Here, the ratio of behavior $B_{a}$ relative to behavior $B_{b}$ depends on the the ratio of the availability of reinforcers $(R)$ for each operandum; the ratio of two bias terms $\kappa_{a}$ and $\kappa_{b}$, each one pushing the animal toward its own option; and the sensitivity parameter $s$ that describes the degree to which subjects change behavior as relative reinforcement changes. In the event that more than two choice alternatives are under consideration, animals allocate their foraging effort on any option in proportion to all the others. Extending this equation to every operandum $i$ (as described by Jensen, 2014b) gives:

$$
\frac{B_{i}}{G M(\boldsymbol{B})}=\frac{\kappa_{i}}{G M(\boldsymbol{\kappa})}\left(\frac{R_{i}}{G M(\boldsymbol{R})}\right)^{s}
$$

Here, $G M(x)$ refers to the geometric mean of $x$. This formulation is arithmetically identical to the two-item case, and extends the same ratio-based relationship symmetrically to all stimuli when three or or more alternatives are considered.

It is no exaggeration to say that thousands of papers have been written on this model, adding parameters or modifying its form to improve the goodness of fit. Nevertheless, its descriptive power is limited to summaries of behavior, counts of responses accumulated over the course of a session or tens of sessions. More granular studies suggest that animals match locally, and change their behavior almost as soon as the reinforcement contingencies change (Gallistel et al., 2001). The next logical step for the study of matching is to consider process models that evaluate individual choices.

In our view, one of the successes of modern reinforcement learning is the actor-critic architecture (Barto et al., 1983), which models behavior as a two-step process. First, the agent (or organism) takes an action using its action policy (e.g. "pick whichever operandum has the highest estimated value"). Then, the agent critiques its action, updating the value function for that action, based on how well it worked (e.g. "that choice yielded less food than expected, so reduce its estimated value accordingly"). This two-step process is almost always implicit in behavior analysis experiments, sometimes being lumped into a single step. There is a subtle reason why: In behavior analysis, response rates are taken to reflect associative value; however, associative value does not (necessarily or uniquely) cause response rates. Furthermore, measuring responding as a rate averages over prior circumstances. The events immediately preceding a choice may make that choice much more or less likely.

Thus, despite consistently fitting session-level data, the matching law equations (Equations 2 and 3) have revealed very little about the decision processes that give rise to them. This is especially true in the case of Equation 3, because as the number of operanda increases, the potential complexity of the 


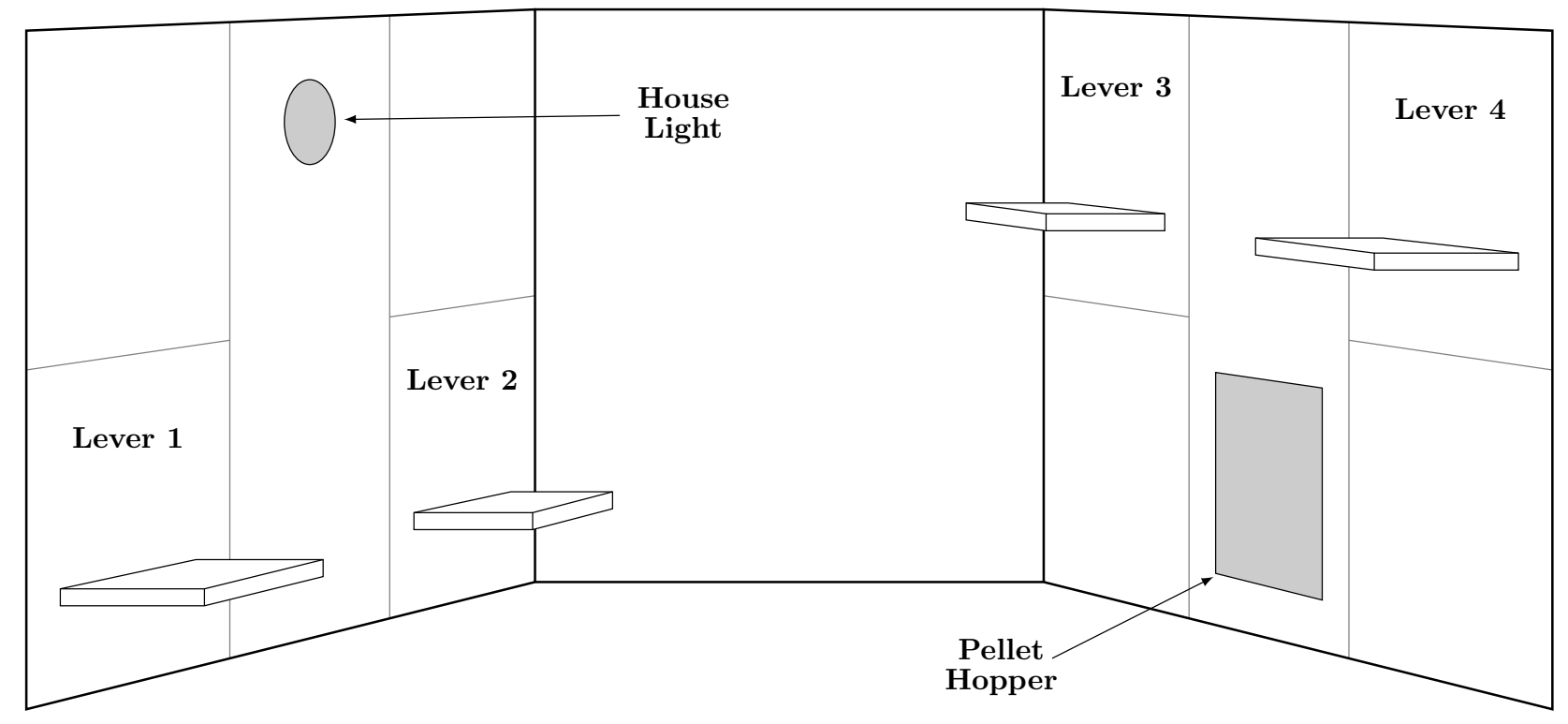

Figure 2. Operant chamber layout. Lever were fixed in place and could not retract. The front and back walls were clear plexiglas, while the walls surrounding the operanda were made of steel. See Jensen (2014a) for more information.

resulting behavior grows exponentially. With two alternatives, the animal can either stay with their current choice or switch to the alternative. Assuming the animal switches, it now has the option, again, to stay or switch. In total, there are four $\left(2^{2}\right)$ possible transitions from one alternative to another. Adding one more alternative increases the total list of possible transitions to nine $\left(3^{2}\right)$; adding still another increases the list to sixteen $\left(4^{2}\right)$. To explore this complexity, Jensen (2014a) collected data from rats in operant chambers that contained anywhere from two to eight operanda. For our example, we will focus on data collected from four-operandum sessions, whose configuration is depicted in Figure 2. Reinforcers were available probabilistically, in a manner that resembles a variable interval schedule.

The apparatus is shown in Figure 2. Two of the levers are close to the ground (and thus easily operated), but are far away from the pellet dispenser. The other two levers are close to the dispenser, but are suspended about halfway up the box, and are harder to operate.

Reinforcement learning provides the computational tools that make process models much more straightforward to evaluate. Below, we give a fully worked example of how to apply an RL model that closely resembles the Rescorla-Wagner model. In doing so, we reveal factors that govern how rats make decisions, but that are concealed by Equation 2.

The first step in building our process model is to specify the value policy: How do subjects update their estimates of value based on feedback from the environment? A simple RL approach to this problem is to use $Q$-learning (Watkins and Dayan, 1992), similar to the Rescorla Wagner model, and inspired by the Widrow-Hoff algorithm (Rescorla-Wagner's machine learning predecessor). In $Q$-learning, a "quality” is calculated at a time $t$ for each available action $a_{t}$ in the current state $s_{t}$; this quality is denoted by $Q\left(a_{t} \mid s_{t}\right)$. When an action is taken, time advances to $t+1$, and the obtained outcome $r_{t}$ and the resulting state $s_{t+1}$ are taken into account. These are then used to retroactively adjust the previous value of $Q$ :

$$
Q\left(a_{t} \mid s_{t}\right) \leftarrow Q\left(a_{t} \mid s_{t}\right)+\alpha\left[r_{t}+\gamma \cdot \max \left(Q\left(a l l \mid s_{t+1}\right)-Q\left(a_{t} \mid s_{t}\right)\right)\right]
$$

Here, $\alpha$ is the learning rate (ranging from 0.0 to 1.0), which governs the degree to which $Q$ is altered 
moment-by-moment. The outcome $r_{t+1}$ may have any positive or negative value, but typically reflects the amount of reinforcement given. Finally, the $\gamma$ parameter discounts "optimal future potential earnings" (i.e. the best that can be expected, given all available actions) from state $s_{t+1}$. It forces a trade off between the future actions and the current prediction error. This effectively penalizes behaviors that put the agent in immediate danger (e.g. jumping off a cliff in pursuit of prey). In the present experiment, every operandum has the potential to yield a reward, and all rewards are of equal size. Consequently, the maximum potential earnings in state $s_{t+1}$ are always the same, and so are not informative. To present as simple a case as possible, we set $\gamma$ to zero, yielding the simplest possible case:

$$
Q\left(a_{t} \mid s_{t}\right) \leftarrow Q\left(a_{t} \mid s_{t}\right)+\alpha\left(r_{t}-Q\left(a_{t} \mid s_{t}\right)\right)
$$

This is arithmetically close to the Rescorla-Wagner updating rule, but the emphasis on considering both state and action is crucial to simulating behavior in complex environments. The Rescorla-Wagner model views $\mathrm{Q}$ as the strength of an association (either S-S or S-R), but RL views it as the quality of taking an action in a given state. For simplicity, we will consider $s_{t}$ to correspond to the subject's location in the operant chamber following each precious response. This captures a detail of behavior that many implementations of the RW model miss: That choosing Lever 1 may be much more likely if the organism is already standing next to it than if it has to travel across the chamber to do so.

Because Equation 5 only specifies the value policy, our model still needs rule for converting the value of $Q$ in any given state into a policy for selecting which action to take. The agent could, for example, always select the action with the highest expected return, or could ignore $Q$ altogether and respond randomly. A popular option is to make random choices whose probability is relative to $Q$, with an exponential parameter similar to $s$ in the matching law. To avoid confusion, we will call this " $Q$-sensitivity" parameter $\theta$, and use it to obtain a value $V_{i}$ for each operandum $i$ :

$$
V_{t_{i}} \leftarrow \theta \cdot Q\left(i \mid s_{t}\right)+K\left(i \mid s_{t}\right)
$$

In addition to $\theta$, a bias term $K$ is introduced, which like $Q$ is conditional on the agent's current state. Rather than understanding bias in terms of the properties of an operandum, this " $Q$-bias" is better understood as a bias for or against a particular action, in this example, a particular transition from one place to another in the operant chamber.

Armed with a value for each operandum, the probability of selecting an operandum when in any given state is given by the softmax rule:

$$
p\left(i \mid s_{t}\right)=\operatorname{softmax}\left(\boldsymbol{V}_{\boldsymbol{t}}\right)=\frac{\exp \left(V_{t_{i}}\right)}{\sum_{j} \exp \left(V_{t_{j}}\right)}
$$

Behavior of this kind can be presented compactly as a matrix, such that each row corresponds to a state, and each column to one of the actions contingent on that state. This effectively casts the behavior as a Markov Decision Process (MDP) (Sutton and Barto, 1998). It also provides explicit rules for how to generate a continuous series of choices as feedback comes in. Provided we can obtain parameter estimates, we can not only describe a subject's past behavior, but can potentially simulate new behavior in previously-untested experimental manipulations.

For many behavior analysts, learning to use the tools that make fitting parameters possible will be the most difficult transition to make. The benefits of doing so are enormous because a wide range of 
new experimental questions become available. To fit the parameters that appear in Equations 5 and 6 (namely, $\alpha, \theta$ and each bias term $K\left(i \mid s_{t}\right)$, a total of 22 terms), we used the Stan programming language (Carpenter et al., 2017), the code for which is provided, and annotated, in the appendix, as well as from an Open Science Foundation repository ( https://osf.io/rt54h/ ). Running this analysis will yield a posterior distribution for every parameter simultaneously (including any implicit covariation that might exist among parameters), permitting us to make inferences about any descriptive statistic we might wish to examine, as well as to perform whatever simulation or inference we might wish to undertake.

Overall, this approach appears very different from generalized matching. To understand the connection, it is important to distinguish matching as a process from matching as an outcome. Generalized matching (as in Equations 2 and 3) can really only be said to describe an outcome, since its fundamental data come in summary form. While some have hypothesized that matching is also a process (i.e. that organisms actively estimate proportions of reinforcement and steer responding to correspond with it) (Hinson and Staddon, 1983; Prelec, 1984), an analysis of summary data can't firmly establish this.

What $Q$-learning is interested in, however, is not an estimate of the the proportion of reinforcement (i.e. $\frac{R_{i}}{\sum \boldsymbol{R}}$ ), but rather an estimate of the expected value of a reinforcers $R_{i}$ per response $B_{i}$ (i.e. $\frac{R_{i}}{B_{i}}$ ). Like the Rescorla-Wagner model, $Q$-learning (Equation 5) takes a weighted average of the outcomes associated with a particular response, with recent events counting more than events long past. The transformation of the value function (Equation 6), aside from introducing bias term, modifies the estimates of $Q$, exaggerating the contrast when $\theta$ is greater than one and reducing the contrast when $\theta$ is less than one. Finally, the softmax rule (Equation 7) probabilistically chooses which action to take, favoring responses with higher values (and therefore, all else being equal, favoring the response with the largest $\frac{R_{i}}{B_{i}}$ ratio). When an estimate of $\frac{R_{i}}{B_{i}}$ based on recent trials is large relative to other operanda, the algorithm should favor responding to $i$.

In variable ratio schedules, $\frac{R_{i}}{B_{i}}$ is invariant from one trial to the next, so $Q$-learning will always favor the richer alternative and, given a large value of $\theta$, will reliably choose the better option almost exclusively, which is optimal. Variable interval schedules, however, have the feature that excessive responding to an alternative lowers its $\frac{R_{i}}{B_{i}}$ ratio, whereas infrequent responding increases it. Killeen (2015) describes variable interval schedules as "contraptions" that steer behavior using negative feedback loops, and it is this negative feedback that $Q$-learning can exploit. Over time, it steers behavior toward whichever response alternative has the highest value of $\frac{R_{i}}{B_{i}}$, and because excessive responding to an alternative lowers that fraction, the behavior ultimately reaches some balancing point. Generalized matching is a description of the resulting equilibrium.

Figure 3 shows boxplots of the posterior distributions for each of the model parameters for a single subject. The filled circle gives the mean of the posterior distribution, the box gives the $80 \%$ credible interval, and the error bars give the $99 \%$ credible interval. The parameters for this subject reveal a few things about its behavior, at least respect to this model. First, the subject updates its model relatively slowly, as evidenced by the small value for $\alpha$. A good rule of thumb is that $\frac{1}{\alpha}$ gives a rough idea of how many trials are meaningfully influencing the algorithm's current estimate of $Q_{i}$; in this case, something akin to the last 100 trials. Put another way, each new reinforcement only contributes about $\frac{1}{100}$ to the estimate of $Q$. This is as expected: When schedules of reinforcement change only occasionally (in this case, once every two weeks), it is routine for subjects to adapt slowly to new schedules (Gallistel et al., 2001).

On its face, $\theta$ seems like it should correspond to the sensitivity parameter $s$ in the generalized matching equation. If this were true, then a value for $\theta$ that is greater than 1.0 might come as a surprise, since rodents routinely undermatch. Indeed, when the behavior of this subject was analyzed using the generalized matching law, Equation 3 (as reported in Jensen, 2014a), the very same subject had a sensitivity of 

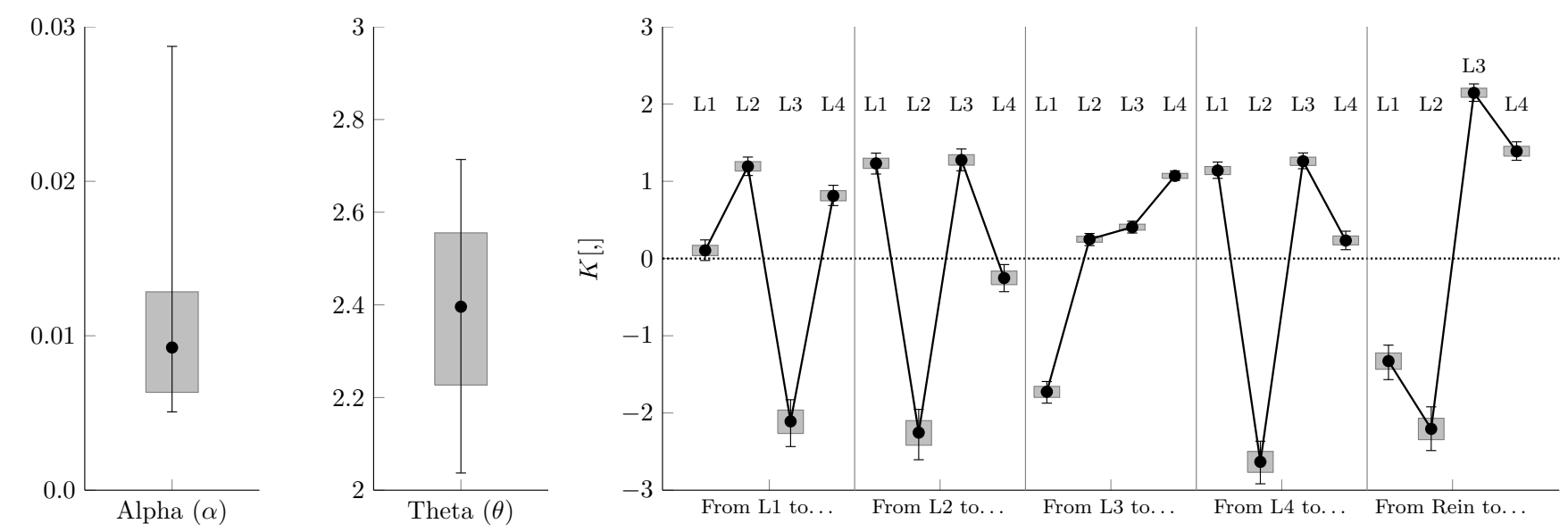

Figure 3. Parameter estimates for the value function $(\alpha)$ and the action policy $(\theta$ and $K[]$,$) for one subject. Points represent$ posterior means, whereas the boxplots depict the $80 \%$ and $99 \%$ credible intervals for the estimates. Values of $K[$,$] were$ centered at 0.0 for each tetrad of parameter values. If a subject's position in the chamber during a prior trial had no impact, then all values of $K[$, ] would be expected to have a value of 0.0 .

$s=0.273$, substantial undermatching. The difference lies in understanding that $\theta$ does not relate to proportions of reinforcement, but instead relates to estimates of expected value. The softmax action policy gives the probability of taking any particular action. With two actions, the softmax action policy resembles a logistic, S-shaped, function that specifies the relationship between the difference in $Q$-values on the $\mathrm{x}$-axis and the probability of choosing that action on the $\mathrm{y}$-axis. The parameter $\theta$ specifies the slope; it specifies how sensitive the rodent is to the difference in the $Q$-values, which change over time as a function of both behavior and the changing schedules.

A high value of $\theta$ means that, at any given moment, a subject strongly favors whichever alternative has the largest current estimate of $\frac{R_{i}}{B_{i}}$. As $\theta \rightarrow \infty$, the behavior tends toward exclusive choice of the current highest estimate. However, because negative feedback from the schedule drives $\frac{R_{i}}{B_{i}}$ down when it is selected too often. Consequently, even though a subject highly favors best ratio, the schedule's negative feedback will cause the subject to alternate between the operanda over the course of the session. There is no conflict between a low value of $s$ and a high value of $\theta$, since the former describes an overall outcome, whereas the latter describes the process of decision-making. This discrepancy has immediate implications for learning theory: It is often said, for example, that rodents are relatively insensitive to ratios of reinforcement (due to their consistently low values of $s$ ), but it may be that rodents are highly sensitive as they are making choices (i.e. display high values of $\theta$ ), and that it is the overall summary measures of their behavior that are insensitive.

The emphasis on process reveals another factor that likely contributes to low values of $s$ : strong biases in response-to-response transitions. Jensen (2014a) reports that subjects who display strong biases in how they transition from one operandum to another tend to display lower sensitivities, because doing so ensures they will spend some time at convenient alternatives, even if those alternatives have a low rate of reinforcement. Jensen (2018) takes this approach a step further, arguing that responses immediately following reinforcer delivery should be considered separately, because an animal's bias depends on their position in the chamber at the time of choice. As in that study, the current example shows powerful transition biases, including following reinforcement. When considering the scale of these biases, keep in mind that $K$ is measured in log units. A 4-point difference in preference for Lever 3 over Lever 2 following reinforcement would mean that, all things being equal, the subject is $\exp (4)=54.6$ times more 
likely to choose Lever 3 than Lever 2.

The rightmost panel of this figure shows what happens immediately after the rat is reinforced (i.e. when it is standing at the hopper). From here, the rat is must more likely to move to Levers 3 or 4 , which are directly above the Pellet Hopper; and much less likely to move to Levers 1 or 2 on the opposite wall. The other panels in the figure show where the rat is likely to go, given where it currently is (which lever it just pressed). The first panel, for example, shows that after the rat presses Lever 1, it moves to Levers 2 or 4, but rarely to Lever 3. That is, it moves laterally, either across the box, or along the back wall, but not diagonally across the chamber's open middle space.

A major advantage of building RL models such that both the value function and the action policy are explicitly defined is that one can use the resulting models to generated simulated behavior. Such data can be used to collect pseudo-pilot data to explore what the effects of unperformed experimental designs might be prior to investing time and living subjects on the problem. It can also be used in the design of experiments to perform power analyses and discover the sample sizes and session lengths needed to obtain reliable effects. Such models can potentially be used in an education context, providing an open-source alternative to Sniffy the Virtual Rat (Alloway et al., 2011). Finally, and most importantly, the model used to describe a subject can subsequently be compared directly to that subject, allowing discrepancies between model and subject to be explored in a more granular fashion.

Baum (2002) suggested the need for a paradigm shift — he called it 'from molecular to molar' — in which an animal's behavior was guided by its molar time allocation decisions rather than its molecular moment-to-moment decisions to press a lever. Animals don't decide to run on a wheel for a quarter turn, and then another quarter turn, and so on. They instead run on a wheel for a specific amount of time, after which it will be time to exhibit some other behavior. Under this molar view, each press, or quarter turn, doesn't matter; it isn't what reinforcement selects for. The alternative view at the time was that each discrete press was guided by response strength, which is selected for by reinforcement. At the heart of the argument, which we are sympathetic to, is a disagreement over what reinforcers acts on. On the one hand, they act to strengthen associations that guide individual responses. On the other hand, they act to readjust the animal's time allocation strategy, which guides individual responses.

One might be tempted to (mis)characterize our position as molecular within this debate because we fit each response using a model of reinforcement that seeks to increase or decrease the quality of each action, similar to response strength. This is not our position. Our position is that, regardless of what reinforcement selects for, computational tools built by computer scientists are becoming increasingly necessary (or at least useful) when building precise models of behavior. If the reader is so inclined, we encourage modification of our model - the code is given in the appendix - to explain and predict the decision at a different choice point: even in "molar" time allocation models of performance, the animal make one choice after another, sequentially, whatever process governs when it moves on to the next activity. A molar approach might be to focus on how the value function evolves over time. A molecular approach might be to focus on how the action policy is implemented. Whichever approach such a modification takes, its persuasiveness should be judged chiefly on how much better is explains and predicts behavior.

\section{What Can Behavior Analysis Do for Computer Science?}

For a statistical algorithm to do its work, it needs data to act on. Often, it needs intervening variables, measurements or settings that help the algorithm transform the input into the output. For example, the standard deviation is the consequence of a statistical algorithm that takes in an array of numbers, computes the mean as an intermediate step, and spits out a single number that represents the dispersion of the input vector with respect to that mean. The Q-learning algorithm takes as its input the choice and reinforcement outcome of the previous state (or trial), updates Q-values (and computes prediction errors) 
as an intermediate step, which enables the action policy to spit out a probability of taking a particular action. Without the input, the algorithm is sunk before it leaves the harbor. Without the output, the algorithm can't predict new behavior. And without the intermediate steps, it can't explain that behavior. Absent any of these, the algorithm is useless.

Behavior Analysts are in a position to help define what the input, output, and intermediate steps should be. We describe three candidates for the input in this paper: the response and reinforcer history (what we modeled here), the response rate and reinforcement rate history, or the time allocation and outcome history. Which one of these reinforcement acts on is unclear in part because they are interrelated in most experiments. Both the response rate and time allocation depend on the timescale over which each lever press, each choice, is made. The candidates for the output seem obvious, but they are not. The obvious choice is to predict what the algorithm acts on; the response and outcome history should predict the next response and outcome, for example. This is what Q-learning does. But should the model in which reinforcement acts on time allocation predict future time allocation, or should it predict the individual responses that make up how an animal spends its time? We described several candidates for the intermediate steps: animal's form associations, they compute $Q$-values, they estimate reinforcement rate ratios, and they track how time is allocated. Each of these can be modified by reinforcement, that, when combined with the other choices we have to make as researchers, leaves the space of possible models large, but pushes toward a computational framework for explaining and predicting behavior.

\section{Appendix: Annotated Stan Code}

The Stan model used to fit the parameters above uses data from a single subject, with data from multiple sessions concatenated into a single continuous stream of events. The full model takes the following form:

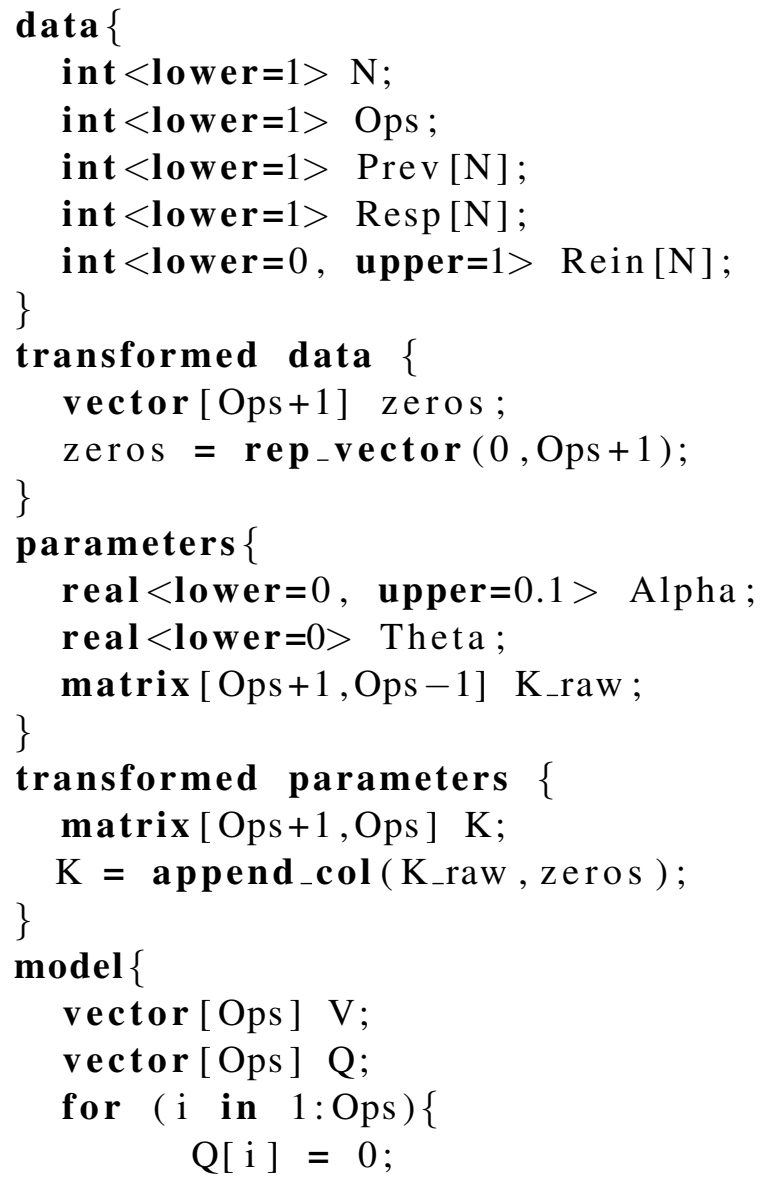




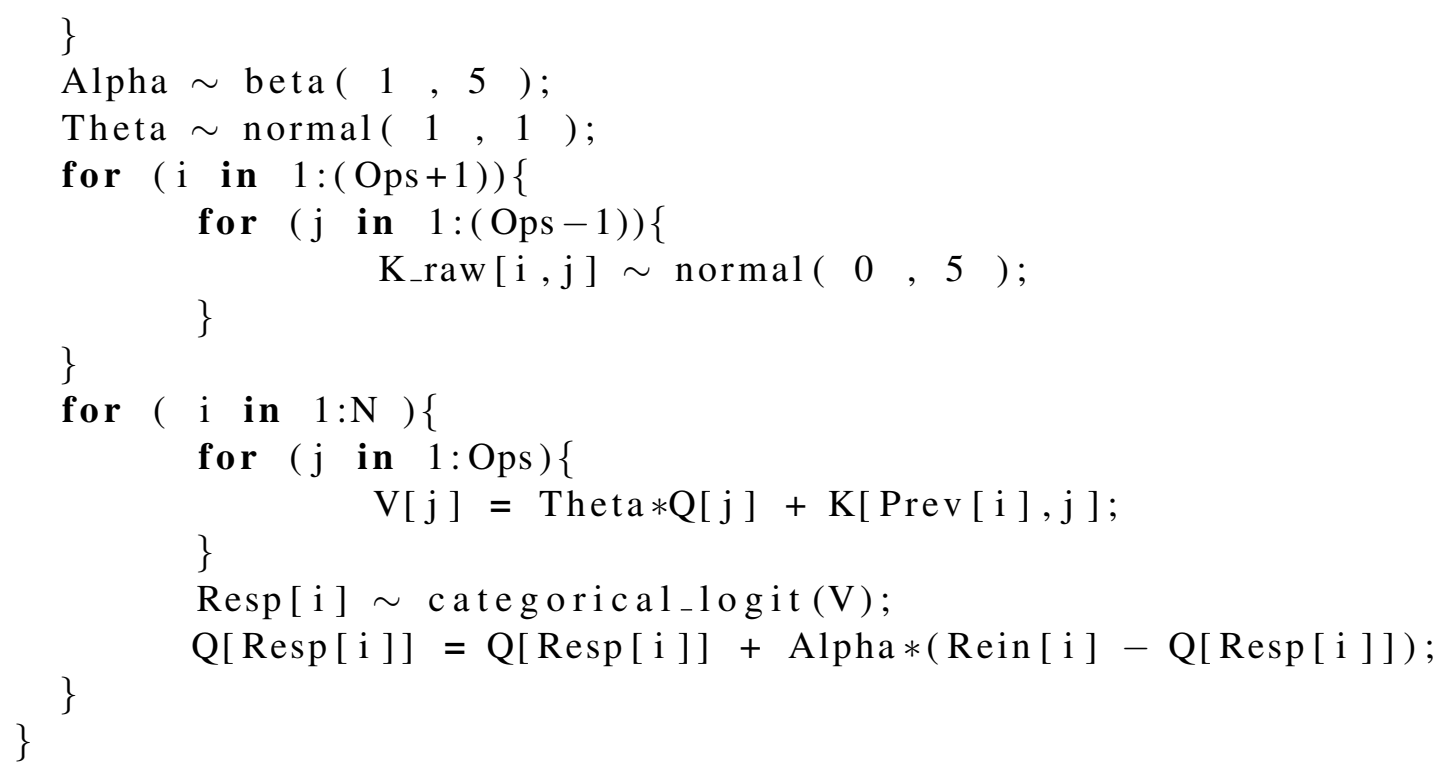

Stan models are divided into "blocks" that are presented in a fixed order. Not every model will require every kind of block. In this case, the first block is the data block, which declares those variables that will be treated as data in this analysis. In Stan, it is important to declare as much as possible about the model in explicit terms.

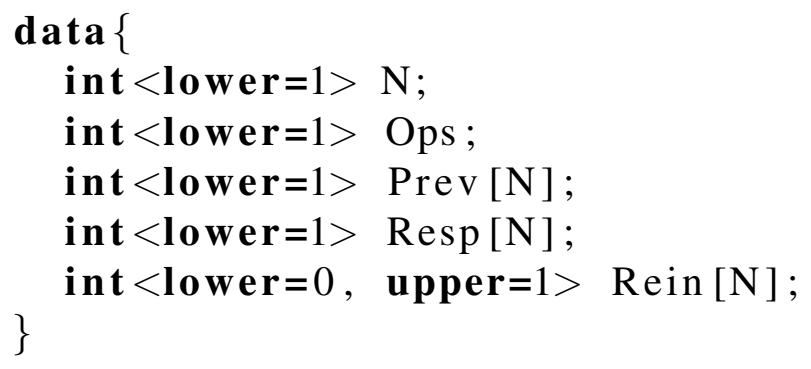

The first two declared values are $\mathrm{N}$ (the number of observations in the data) and Ops (the number of operanda in the chamber). Both are declared to be integers with a lower bound of 1 . As a result, the model would refuse to run if we were to tell Stan that we had 0 observations (below its lower bound), or that we had 0.25 observations (because that is a real number and not an integer).

Prev, Resp, and Rein are one-dimensional arrays of length N, with each entry corresponding to a particular trial. The $[\mathrm{N}]$ that follows each indicates that they are arrays and specifies their length. These correspond, respectively, to (a) the subject's previous location in the chamber, (b) the subject's response, and (c) whether that trial was reinforced or not. The values of these arrays must be integers, with explicit bounds declared. While it is not always mandatory to set bounds on variables, it is generally good practice because it can help the model run more efficiently. It can also help the analyst detect aberrant observations. For example, some datasets code missing observations as negative numbers, and if these are not removed or recoded appropriately, a lower bound will alert the analyst that impossible values have been included in the analysis.

One of the subtle difficulties in working with the softmax rule (Equations 6 and 7) is that the bias term $\mathrm{K}$ is unitless, and strictly relative. Generalized matching has the same difficulty: in Equation 2, there is no meaningful difference between $\left(\kappa_{a}=1, \kappa_{b}=2\right)$ and $\left(\kappa_{a}=10, \kappa_{b}=20\right)$, since the values of $\kappa_{i}$ are only meaningful relative to one another. Consequently, in order to make an inference about the bias terms, it is necessary to set one of of the terms to an arbitrary value, using it as a reference category. In effect, each row of the matrix $\mathrm{K}$ has one degree of freedom fewer than it has columns. 
With these considerations in mind, consider the following blocks of code:

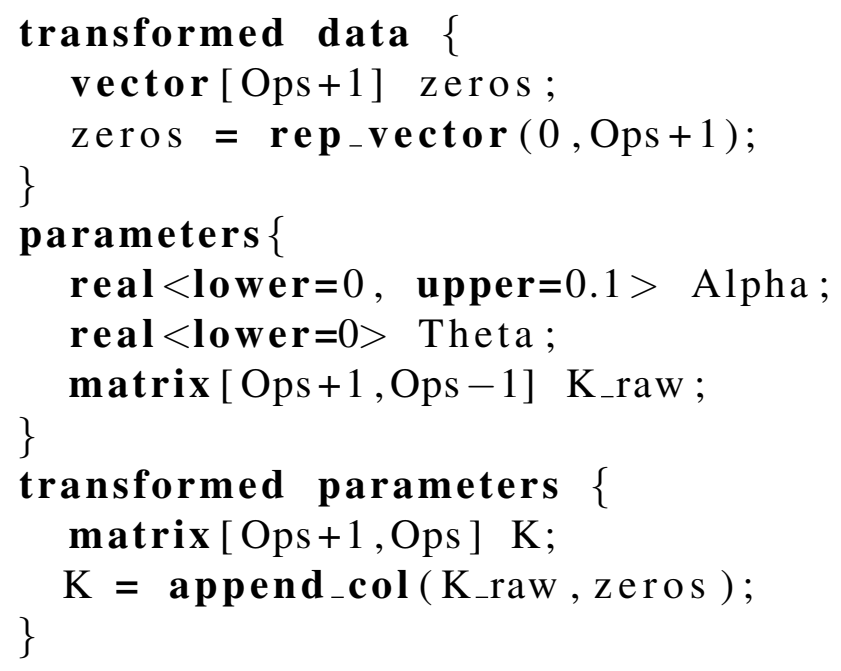

The purpose of these blocks is to set up the parameters Alpha, Theta, and K, which are used to implement Equations 5, 6, and 7. Normally, the parameters block is dedicated to declaring all free parameters about which we would like to make inferences. However, instead of declaring $\mathrm{K}$ in the parameters block, the matrix $\mathrm{K}$ raw is instead declared. $\mathrm{K}$ raw refers to those columns in $\mathrm{K}$ that are free to vary. The transformed parameters block is then used to declare $\mathrm{K}$, by appending a column of zeroes to its right side. This column of zeroes was ready-made in the transformed data block for just this purpose. Henceforth, the values of the rightmost column of $\mathrm{K}$ will be fixed to zero, and the other columns will be allowed to vary.

The model block is where the prior distributions for the parameters are specified and the sampling procedure is implemented. Each of these functions will be considered in turn.

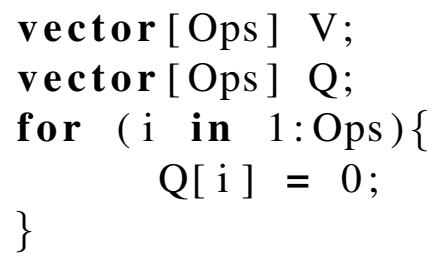

First, the vectors $\mathrm{V}$ and $\mathrm{Q}$ are declared as containers for trial-by-trial value estimates. These are not parameters as such, and are not saved. Since their values depend strictly on the parameters and on the data, their values at any trial can easily be recalculated after the fact. The values of $Q$ are set to zero by default, but their values will rapidly evolve as a function of feedback from the schedule.

Next, the prior distributions for each of the parameters is declared.

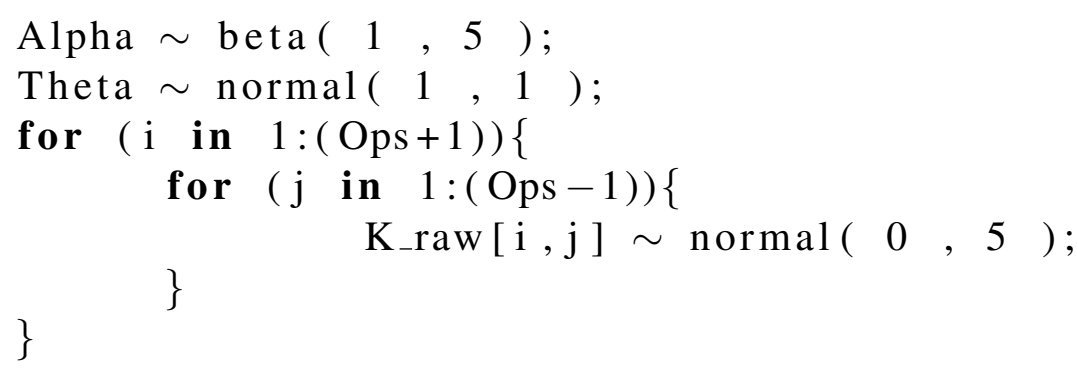

Notice that the ' $\sim$ ' symbol is used instead of the '=' symbol. When $\sim$ appears in these declarations, it should be read as "is sampled from." For example, 'Alpha $\sim$ beta ( 1,5 )' should be read as, "The parameter Alpha is sampled from a beta distribution with shape parameters 1 and 5." Similarly, 
'Theta $\sim \operatorname{normal}(1,1$ )' should be read as, "The parameter Theta is sampled from a normal distribution with a mean of 1 and a standard deviation of 1."

In each of these cases, we have chosen to use a weakly informative prior distribution, so that sampling benefits from regularization. In each case, the distribution covers the full range of values that we consider plausible, but tends to omit values that are implausible. For example, a value of Theta close to zero is plausible if subjects are insensitive to differential probabilities of reinforcement, but extreme negative values (e.g. - 10.0) are very implausible because rodents do not generally strenuously avoid receiving food reinforcers.

Finally, the model block must evaluate the data themselves. For every trial and every operandum, the values of $\mathrm{V}$ and $\mathrm{Q}$ need to be updated, and the likelihood of the observed response choice must be calculated. Because we are fitting a process model, this is done one trial at a time using a loop.

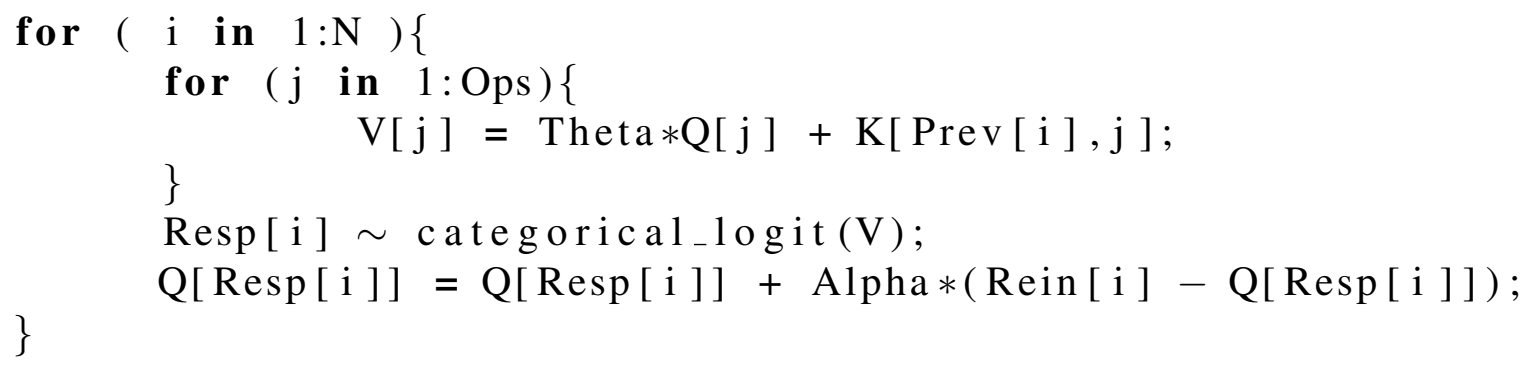

The line ${ }^{\prime} \mathrm{V}[\mathrm{j}]=$ Theta $* \mathrm{Q}[\mathrm{j}]+\mathrm{K}[\operatorname{Prev}[\mathrm{i}], \mathrm{j}]$ ' is simply the implementation of Equation 6, and the line ' $\mathrm{Q}[\operatorname{Resp}[\mathrm{i}]]=\mathrm{Q}[\operatorname{Resp}[\mathrm{i}]]+\operatorname{Alpha} *(\operatorname{Rein}[\mathrm{i}]-\mathrm{Q}[\operatorname{Resp}[\mathrm{i}]])^{\prime}$ is the implementation of Equation 5. Although it is not immediately obvious, the line 'Resp[i] categorical_logit (V);' is actually the implementation of Equation 7. It can be read as, "the likelihood of the response at trial $i$ is sampled from the categorical distribution according to the softmax rule." Another way to write this line is 'Resp[i] categorical ( $\operatorname{softmax}(\mathrm{V})$ ), ' but it is preferable to use the logit construction because it avoids unnecessary exponential transforms that can introduce rounding errors.

Stan uses a particularly efficient Markov chain Monte Carlo (MCMC) algorithm to fit the data. A model specifies the parameter space. In this case, the are 22 parameters $(\theta, \alpha$, and one $K$ parameter for each of 20 possible transitions), so the parameter space has 22 dimensions. The best fitting parameters is a single point in this high-dimensional space. Finding this point, that is, fitting the model to the data, is the same as moving this point around through parameter space, keeping track of how well that point fits the data. Moving the particle around, storing its position after each movement, slowly builds up the posterior probability function. The algorithm Stan uses, "Hamiltonian Monte Carlo," moves the point through this parameter space so that it constructs the joint posterior probability function quickly and efficiently. With these samples, it is a simple matter taking the mean and standard deviation to get the parameter estimates, and its standard error. But more important, we can treat these samples a bit like data, using them to construct any summary measure of the parameters we want. Figure 3 shows the mean of each parameter (black circle), as well as the $80 \%$ credible interval (box) and the $99 \%$ credible interval (whisker) for each estimate. These descriptives are calculated directly from the samples generated by the MCMC sampler. Good introductions to MCMC sampling for parameter estimation are given by Kruschke (2015) and McEltreath (2016).

\section{ACKNOWLEDGMENTS}

The authors owe a debt of gratitude to Peter Balsam and Kathleen Taylor, who oversaw data collection. Additionally, this manuscript would not have arisen were it not for fruitful discussions with the Neurostorm 
group, including Matthew Bailey, David Barack, Erin Kendall Braun, Brittany DeFeis, Christophe Dupré, Emily Feierman, Paul Frazel, Joud Hijazi, Raphael Gerraty, Olivia Goldman, Nuri Jeong, Ben Joffe, Terese Lawry, Peter Mercado-Reyes, Christopher Mezias, eMalick Njie, Elke Schipani, Ben Shababo, Michelle van Tieghem, and Matti Vuorre.

\section{AUTHOR CONTRIBUTIONS}

GJ and DF wrote the manuscript and performed the analyses.

\section{REFERENCES}

Alloway, T., Wilson, G., and Graham, J., editors (2011). Sniffy the Virtual Rat Pro, Version 3.0. Wadsworth, Belmont, CA, USA.

Arcediano, F. and Miller, R. R. (2002). Some constraints for models of timing: A temporal coding hypothesis perspective. Learning and Motivation, 33:105-123.

Azar, M. G., Munos, R., Ghavamzadeh, M., and Kappen, H. (2011). Reinforcement learning with a near optimal rate of convergence. HAL, inria-00636615.

Barto, A. G., Sutton, R. S., and Anderson, C. W. (1983). Neuronlike adaptive elements that can solve difficult learning control problems. IEEE Transactions on Systems, Man, and Cybernetics, SMC-13:834846.

Baum, W. M. (1974). On two types of deviation from the matching law: Bias and undermatching. Journal of the Experimental Analysis of Behavior, 22:231-242.

Baum, W. M. (2002). From molecular to molar: A paradigm shift in behavior analysis. Journal of the Experimental Analysis of Behavior, 78:95-116.

Bellman, R., editor (1957). Dynamic Programming. Princeton University Press, Princeton, NJ, USA.

Bouton, M. E. and Hendrix, M. C. (2011). Intertrial interval as a contextual stimulus: Further analysis of a novel asymmetry in temporal discrimination learning. Journal of Experimental Psychology: Animal Behavior Processes, 37:79-93.

Bush, R. R. and Mosteller, F., editors (1955). Stochastic Models for Learning. Wiley, New York, NY, USA.

Carpenter, B., Gelman, A., Hoffman, M., Lee, D., Goodrich, B., Betancourt, M., Brubaker, M. A., Guo, J., Li, P., and Riddel, A. (2017). Stan: A probabilistic programming language. Journal of Statistical Software, 76:1-32.

Clayton, N. S. and Dickinson, A. (1998). Episodic-like memory during cache recovery by scrub jays. Nature, 395:272-274.

Dally, J. M., Emery, N. J., and Clayton, N. S. (2006). Food-caching western scrub-jays keep track of who was watching when. Science, 312:1662-1665.

Daw, N. D. and Courville, A. C. (2007). The pigeon as particle filter. In Platt, J. C., Koller, D., Singer, Y., and Roweis, S. T., editors, Advances in Neural Information Processing Systems, volume 20.

Fantino, E. (2008). Behavior analysis: thriving, but how about its future? Journal of the Experimental Analysis of Behavior, 89:125-127.

Gallistel, C. R. (2007). Flawed foundations of associationism? comment on machado and silva (2007). Americal Psychologist, 62:682-685.

Gallistel, C. R., Mark, T. A., King, A. P., and Latham, P. E. (2001). The rat approximates an ideal detector of changes in rate of reward: Implications for the Law of Effect. Journal of Experimental Psychology: Animal Behavior Processes, 27:354-372. 
Herrnstein, R. J. (1961). Relative and absolute strength of responses as a function of frequency of reinforcement. Journal of the Experimental Analysis of Behavior, 4:267-272.

Hinson, J. M. and Staddon, J. E. R. (1983). Matching, maximizing, and hill-climbing. Journal of the Experimental Analysis of Behavior, 40:321-331.

Hull, C. L., editor (1943). Principles of Behavior. Appleton-Century-Crofts, New York, NY, USA.

Jensen, G. (2014a). Beyond dichotomy: Dynamics of choice in compositional space (doctoral dissertation). Technical Report DOI 10.7916/D8NC5ZC4, Columbia University.

Jensen, G. (2014b). Compositions and their application to the analysis of choice. Journal of the Experimental Analysis of Behavior, 102:1-25.

Jensen, G. (2018). Choice under conditions of uncertainty: Methods for analyzing the structure of behavior. Psyarchiv Preprints, DOI 10.31234/osf.io/579p8.

Kalafut, K. L. and Church, R. N. (2017). The quantification of behavior in the presence of compound stimuli. Journal of Experimental Psychology: Animal Learning and Cognition, 43:96-93.

Kamin, L. J. (1969). Predictability, surprise, attention, and conditioning. In Campbell, B. A. and Church, R. M., editors, Punishment and Aversive Behavior, pages 279-296. Appleton-Century-Crofts, New York, NY, USA.

Killeen, P. R. (2015). The logistics of choice. Journal of the Experimental Analysis of Behavior, 104:74-92. Kruschke, J. K., editor (2015). Doing Bayesian Data Analysis. Academic Press, London, UK.

Lenat, D. (2008). The voice of the turtle: Whatever happened to ai? AI Magazine, 29:11-22.

Mazur, J. E. (2006). Mathematical models and the experimental analysis of behavior. Journal of the Experimental Analysis of Behavior, 85:275-291.

McEltreath, R., editor (2016). Statistical Rethinking. CRC Press, Boca Raton, FL, USA.

Michael, J. (1980). Flight from behavior analysis (presidential address, aba, 1980). Behavior Analyst, 2:1-22.

Miller, R. R., Barnet, R. C., and Grahame, N. J. (1995). Assessment of the Rescorla-Wagner model. Psychological Bulletin, 117:363-386.

Morris, R. W. and Bouton, M. E. (2006). Effect of unconditional stimulus magnitude on the emergence of conditioned responding. Journal of Experimental Psychology: Animal Behavior Processes, 32:371-385.

Prelec, D. (1984). The assumptions underlying the generalized matching law. Journal of the Experimental Analysis of Behavior, 41:101-107.

Rescorla, R. A. (1971). Variation in the effectiveness of reinforcement and nonreinforcement following prior inhibitory conditioning. Learning and Motivation, 2:113-123.

Rescorla, R. A. and Wagner, A. R. (1972). A theory of pavlovian conditioning: Variations in the effectiveness of reinforcement and non-reinforcement. In Black, A. H. and Prokasy, W. F., editors, Classical Conditioning II: Current Research \& Theory, pages 64-99. Appleton-Century-Crofts, New York, NY, USA.

Ribas-Fernandes, J. J. F., Solway, A., Diuk, C., McGuide, J. T., Barto, A. G., Niv, Y., and Botvinick, M. M. (2011). A neural signal of hierarchical reinforcement learning. Neuron, 71:370-379.

Savastano, H. I. and Miller, R. R. (1998). Time as content in pavlovian conditioning. Behavioural Processes, 44:142-162.

Siegel, S. and Allan, L. G. (1996). The widespread influence of the Rescorla-Wagner model. Psychonomic Bulletin \& Review, 3:314-321.

Silver, D., Hubert, T., Schrittwieser, J., Antonoglou, I., Lai, M., Guex, A., Lanctot, M., Sifre, L., Kumaran, D., Graepel, T., Lillicrap, T., Simonyan, K., and Hassabis, D. (2018). A general reinforcement learning algorithm that masters chess, shogi, and.Go through self-play. Science, 362:1140-1144.

Skinner, B. F. (1950). Are theories of learning necessary? Psychological Review, 57:193-216. 
Solomon, R. L. (1980). The opponent-process theory of acquired motivation: The costs of pleasure and the benefits of pain. American Psychologist, 35:691-712.

Staddon, J. E. R. and Zanutto, B. S. (1998). In praise of parsimony. In Wynne, C. D. L. and Staddon, J. E. R., editors, Models for Action: Mechanisms for Adaptive Behavior, pages 239-267. Lawrence Erlbaum Associates, Mahwah, NJ, USA.

Sutton, R. S. and Barto, A. G. (1981). Toward a modern theory of adaptive networks. Psychological Review, 88:135-170.

Sutton, R. S. and Barto, A. G., editors (1998). Reinforcement Learning: An Introduction. MIT Press, Cambridge, MA, USA.

Thorndike, E. L. (1898). Animal intelligence: An experimental study of the associative processes in animals. Psychological Review, 2:1-109.

Thorndike, E. L., editor (1911). Animal Intelligence. Macmillan, New York, NY, USA.

Wang, J. X., Kurth-Nelson, Z., Tirumala, D., Soyer, H., Leibo, J. Z., Munos, R., Blundell, C., Kumaran, D., and Botvinick, M. (2017). Learning to reinforcement learn. arXiv, 1611.05763v3.

Watkins, C. J. C. H. and Dayan, P. (1992). Q-learning. Machine Learning, 8:279-292.

Widrow, B. and Hoff, M. E. (1960). Adaptive switching circuits. Technical Report 1553-1, Stanford Electronic Laboratories. 